\title{
Multifunctional Protein Materials and Microreactors using Low Complexity Domains as Molecular Adhesives
}

\author{
Lenka Faltova, ${ }^{\dagger, \S}$ Andreas M. Küffner, ${ }^{\dagger, \S}$ Maria Hondele, ${ }^{\ddagger}$ Karsten Weis, ${ }^{\ddagger}$ and Paolo Arosio ${ }^{* \dagger}{ }^{\dagger}$
}

${ }^{\dagger}$ Department of Chemistry and Applied Biosciences, Institute for Chemical and Bioengineering and ${ }^{\ddagger}$ Department of Biology, Institute for Biochemistry, Swiss Federal Institute of Technology, Zurich 8093, Switzerland

\section{Supporting Information}

ABSTRACT: Recent findings indicate that a class of disordered amino acid sequences promotes functional phase transition of biomolecules in nature. Such sequences consist of low complexity domains (LCDs) that are rich in specific amino acids. In this work, we exploit these sequences by conjugating them to soluble globular domains to develop molecular adhesives that enable sensitive, controlled self-assembly of these proteins into supramolecular architectures. In particular, we used the enzyme adenylate kinase and the green fluorescent protein as soluble domains, and we show that the addition of low complexity regions induces the formation of protein particles via a

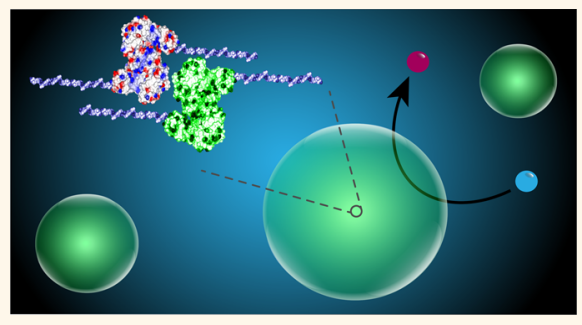
multistep process. This multistep pathway involves an initial liquid-liquid phase transition, which creates protein-rich droplets that mature into protein aggregates over time. These protein aggregates consist of permeable structures that maintain activity and release active soluble proteins. We show that the LCDs dictate specific noncovalent intermolecular interactions and phase properties that are largely independent of the given globular domain. We further demonstrate that this feature, together with the dynamic state of the initial dense liquid phase, allows one to directly assemble different globular domains within the same architecture, thereby enabling the generation of both static multifunctional biomaterials and dynamic microscale bioreactors.

KEYWORDS: low complexity domains, intrinsically disordered proteins, liquid-liquid phase separation, membraneless compartments, protein materials, microreactors, protein self-assembly

$\mathrm{P}$ roteins are intricate macromolecules that are capable of performing specific tasks by interacting with other proteins and biomolecules. In many cases, the activity of proteins requires the self-assembly into complexes and aggregates. Examples in nature include actin filaments, which provide mechanical support in eukaryotic cells, and amyloid structures, ${ }^{1}$ which have been recently associated with a variety of fascinating functions including hormone storage $^{2}$ and biofilm formation. ${ }^{3,4}$

As a result of their high specificity, biocompatibility, and safety, self-assembling protein materials have increasingly found many applications in biotechnology. ${ }^{5,6}$ In this context, the ability to encode specific self-assembly properties by engineering the protein's primary amino acid sequence represents a flexible approach to generate a variety of functional materials. Moreover, the possibility to accurately control the size and the surface chemistry of the protein materials confers tailored properties in terms of mass transport and activity. Examples include peptides that self-assemble into filaments and gels, ${ }^{7-16}$ protein nanocages, ${ }^{17}$ proteinosomes, ${ }^{18}$ and functional amyloids. ${ }^{5}$ These materials have found applications in a variety of fields, including food science, ${ }^{19}$ water treatment, ${ }^{20}$ tissue engineering, ${ }^{21}$ vaccine delivery, ${ }^{22,23}$ biosensors, ${ }^{24}$ and enzyme technology. ${ }^{25}$

Despite these successes, there is still a need to develop strategies to introduce functionalities into the self-organized structures in a simple and flexible manner, for example, if multiple functions are desired within the same structure. Current approaches to generate multifunctional peptide-based materials rely on the post-functionalization of preformed scaffolds, such as nanoparticles and vesicles, with different proteins. This strategy requires the careful control of the conjugation reactions to avoid modifications of protein structure and loss of the material. The development of functional proteins with intrinsic self-association properties would represent an attractive alternative to simplify the aforementioned strategy and convey varying functionalities into a single structure.

In this work, we create chimera proteins that contain a functional, globular domain, responsible for the desired

Received: June 6, 2018

Accepted: September 14, 2018

Published: September 14, 2018 

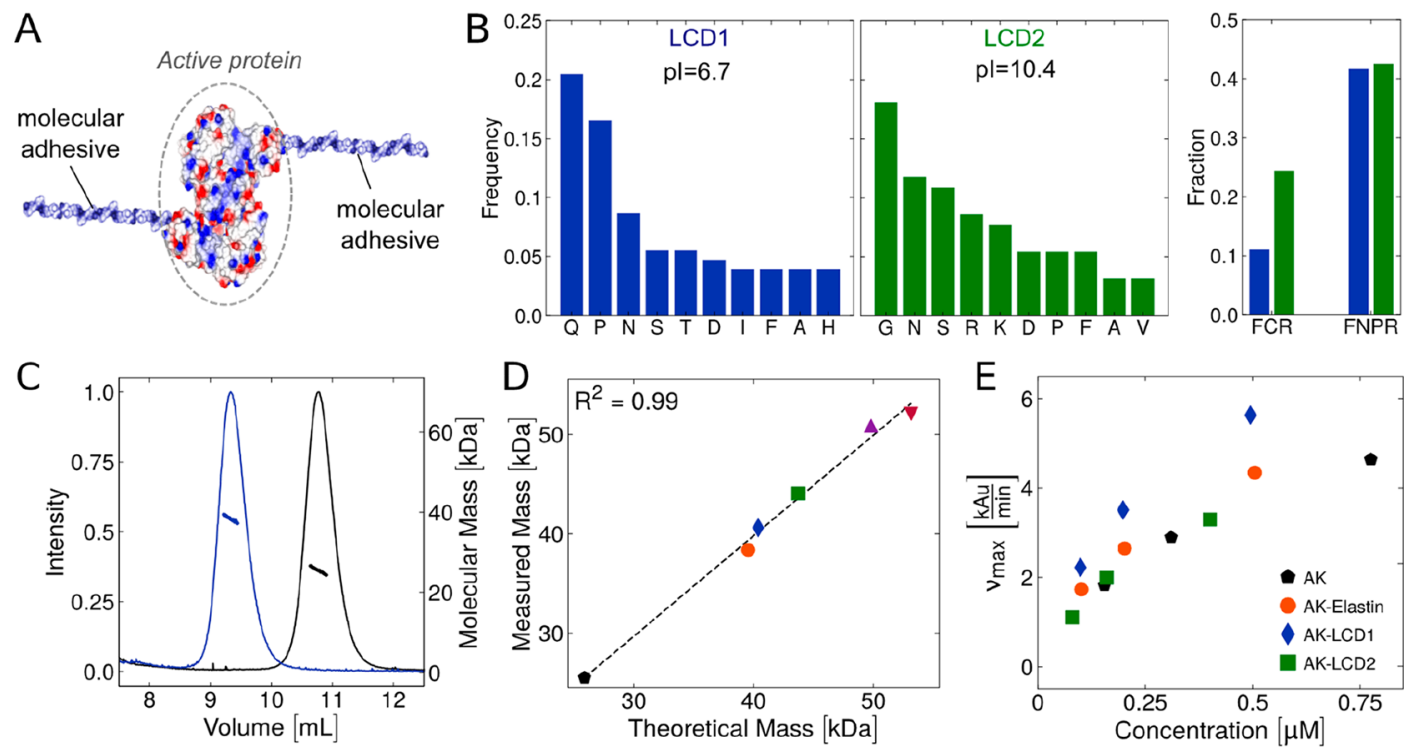

Figure 1. Synthesis and characterization of active chimera proteins. (A) Illustration of the chimera proteins developed in this work in which globular proteins are conjugated with low complexity domains which act as molecular adhesives. The enzyme AK and the GFP have been conjugated with LCDs encoding self-assembling behavior (see Table S1). (B) Amino acid composition and main physiochemical properties of the LCDs including the fraction of charged and nonpolar residues (FCR and FNPR, respectively). pI is the isoelectric point of the proteins. (C-D) Characterization of the molecular weight of the expressed chimera proteins by size exclusion chromatography coupled with multi-angle light scattering: (C) representative chromatograms of AK (black line) and AK conjugated with LCDs originating from Dhh1 (blue line); (D) theoretical versus measured molecular weights of AK (solid $\triangle$ ), AK conjugated with LCDs originating from elastin (๑), Dhh1 $(\downarrow)$, and Dpb1 ( $)$ and GFP conjugated with LCDs originating from Dhh1 $(\boldsymbol{\Delta})$ and Dpb1 $(\nabla) .95 \%$ two-sided confidence intervals are smaller than the markers. (E) Activity of AK and AK chimera proteins evaluated with a fluorometric adenylate kinase activity assay. The conjugation of molecular adhesives has no significant effect on the enzyme activity.

bioactivity, and an intrinsically disordered domain, which acts as a molecular adhesive and enables highly sensitive, controlled self-assembly. This concept has been recently introduced using fragments derived from the hydrophobic protein elastin ${ }^{26,27}$ and with prion-like domains forming amyloid scaffolds. ${ }^{22,25}$ In this work, we apply this principle by exploiting sequences from proteins that have recently been discovered to undergo in vivo phase transition processes associated with the formation of cellular compartments. ${ }^{28-34}$ These motifs are intrinsically disordered and are commonly referred to as low complexity domains (LCD) or low complexity regions (LCR). Such sequences typically contain a high fraction of charged and polar amino acids, such as glutamine, asparagine, serine, arginine, and lysine, and a low fraction of hydrophobic residues. ${ }^{35}$ Furthermore, these motifs are also enriched in residues which disrupt secondary structure, such as proline and glycine. $^{35}$ In these sequences, multivalent attractive interactions between side chains are mediated by specific residues and the highly extended nature of these proteins in aqueous environments. This positive energetic contribution counteracts the entropic loss associated with demixing and consequently leads to liquid-liquid phase separation of protein solutions.

The advantages of these LCDs are two-fold. First, these domains offer the exciting possibility to accurately induce a variety of specific intermolecular interactions using sequences of simple composition. Second, the attractive interactions mediated by such molecular adhesives can induce the formation of a variety of protein-rich phases, ranging from liquid to gel to solid. This high degree of flexibility presents an opportunity to design materials with desired biophysical properties, which are highly sensitive to external stimuli. It is becoming increasingly apparent that nature exploits this ability to induce controlled phase separation of proteins and nucleic acids in order to generate specific membraneless bodies which coordinate reactions in space and time. ${ }^{33,36-38}$

Here, we create chimeric proteins in which we attach LCDs to a globular domain to create a hybrid molecule that maintains the functionality of the globular domain, while mimicking the co-localization behavior of the disordered regions that are found in nature. We show that these molecules undergo liquid-liquid phase transitions to form droplets which mature into protein aggregates and solid particles over time. Interestingly, we show that the dynamic state of the initial dense liquid phase allows one to manipulate structural properties that are maintained in the mature solid state. We demonstrate that this strategy enables the development of protein particles for prolonged release of active proteins as well as microreactors and multifunctional biomaterials, in which different proteins can be directly recruited within the same structure.

\section{RESULTS AND DISCUSSION}

Synthesis, Characterization, and Activity of the Chimera Proteins. We considered the green fluorescence protein (GFP) and the enzyme adenylate kinase (AK) as model globular proteins. We conjugated these two proteins at both the $\mathrm{N}$ - and the $\mathrm{C}$-termini with different LCDs, each with different compositions and physicochemical properties (Figure $1 A)$. Indicated as LCD1 and LCD2, these molecular adhesives are derived from the sequences of the DEAD-box proteins Dhh1 and Dpb1, respectively. In their native biological context, Dhh1 and Dpb1 have been shown to undergo liquid-liquid phase separation associated with the formation of processing bodies (P-bodies) in yeast ${ }^{32}$ (Weis et al., in preparation). 


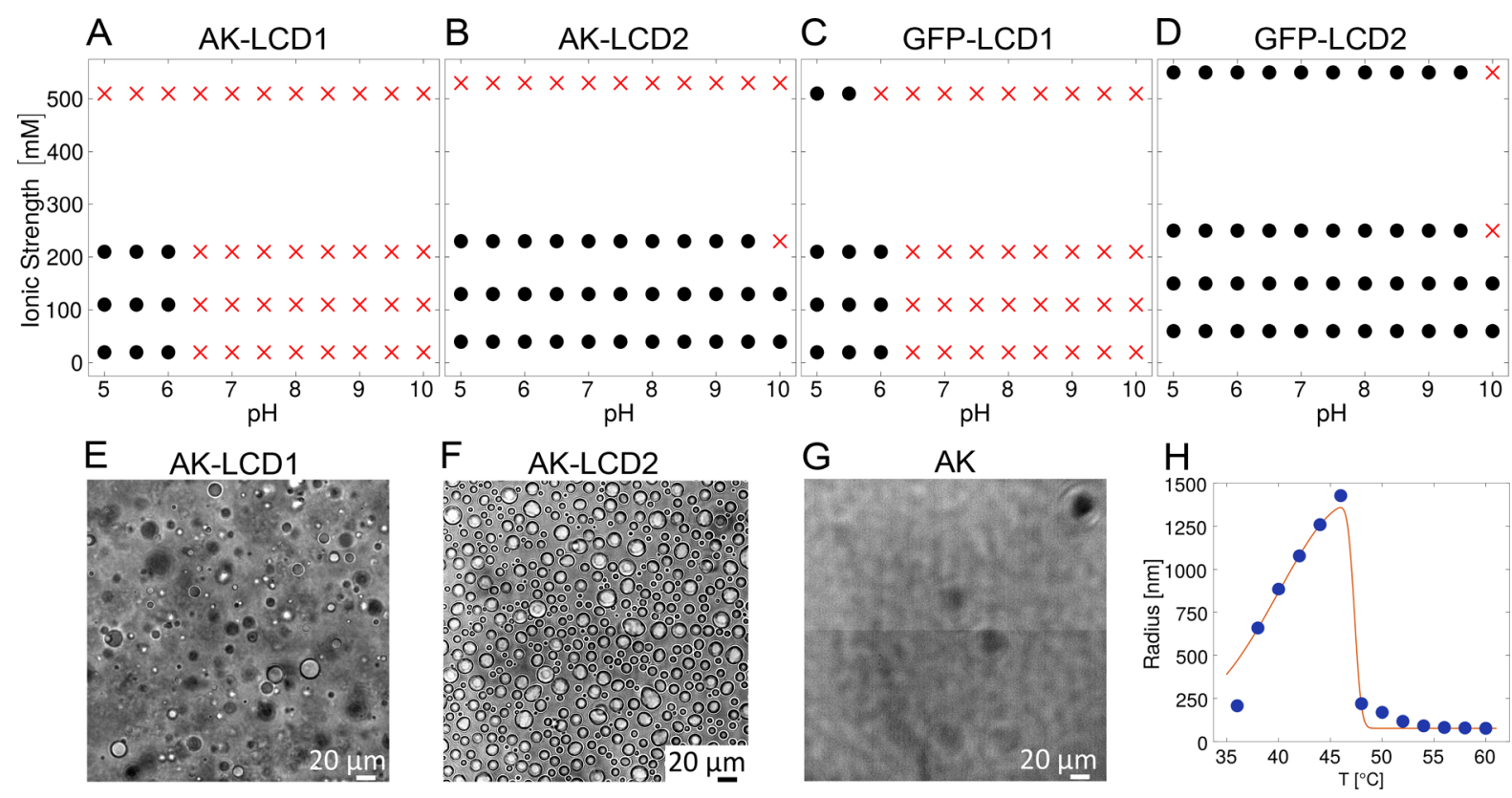

Figure 2. Molecular adhesives encode specific self-assembly of the chimera proteins. Phase diagrams of AK conjugated with LCDs originating from Dhh1 (LCD1) (A) and Dpb1 (LCD2) (B) and of GFP conjugated with LCDs originating from Dhh1 (C) and Dpb1 (D). Circles and red crosses indicate the presence and the absence of phase separation, respectively, as detected by microscopy and DLS (see also Figures S6-S7). The analysis was performed at room temperature. Protein concentrations were: AK-LCD1, 25 $\mu$ M; AK-LCD2, $20 \mu$ M; GFPLCD1, $25 \mu \mathrm{M}$; GFP-LCD2, $5 \mu \mathrm{M}$. Representative microscope images of solutions of AK (E), AK conjugated with LCD1 (F, pH 5.0, no salt), and conjugated with LCD2 (G, pH 8.5, no salt). (H) Estimation of upper critical solution temperature of AK-LCD2 from DLS measurement.

The full sequences of the expressed chimera proteins are summarized in Table S1, and the main physiochemical properties, such as hydropathy, amino acid content, fraction of charged and nonpolar residues, are shown in Figure $1 \mathrm{~B}$ and Figure S1. Both LCD1 and LCD2 contain a high fraction (40\%) of nonpolar residues, particularly glycine and proline, although several charged and polar residues are common throughout the sequences. Specifically, both LCDs share polar residues such as asparagine and serine, and LCD2 contains a high number of the positively charged residues arginine and lysine.

We expressed the chimera proteins in Escherichia coli and characterized their purity and molecular weight by SDS gel electrophoresis (Figure S2) and size exclusion chromatography coupled with a multi-angle light scattering detector (MALS). A representative chromatogram and the measured molecular weights are shown in Figure 1C,D, respectively, and the full set of chromatograms is reported in Figure S3. The experimental molecular weights measured by MALS are in excellent agreement with the theoretical values (Figure 1D). Moreover, to verify that the activity of $\mathrm{AK}$ is not significantly altered by the conjugation with the LCDs, we performed a fluorometric adenylate kinase assay which measures the conversion of the substrate adenosine diphosphate into the fluorescent product (Figure 1E and Figure S4).

Molecular Adhesives Encode Specific Self-Assembling Behavior. We next characterized the phase behavior of the expressed proteins by optical microscopy and dynamic light scattering (DLS). We investigated phase separation at varying salt concentrations and $\mathrm{pH}$ values at room temperature (Figure 2). As expected, the globular proteins AK and GFP without LCDs remained soluble under each condition (Figure S5). In contrast, the fusion proteins containing the LCD1 and the LCD2 domains exhibited phase separation under varying conditions of $\mathrm{pH}$ and salt concentration (Figure $2 \mathrm{~A}-\mathrm{D}$ and Figure S6). The phase transition process was confirmed by the presence of an upper critical solution temperature as measured by DLS (Figure $2 \mathrm{H}$ ).

Notably, the same globular protein conjugated with different LCDs showed a varying solubility as a function of ionic strength and $\mathrm{pH}$, while different proteins (GFP and $\mathrm{AK}$ ) conjugated with the same LCD shared similar phase behaviors. These results not only demonstrate that LCDs can induce phase separation but also that different LCDs can be used to tune specific interactions. Despite the dominant effect of LCD conjugation, different proteins (AK and GFP) conjugated with the same sequence exhibited subtle differences in the phase diagram (Figure 2B,D), indicating that the globular domain can further modulate intermolecular interactions.

To confirm the absence of hydrophobic effects, we generated chimera proteins with a hydrophobic LCD derived from elastin, following a strategy that has been recently proposed in literature (Figure 1D). ${ }^{27}$ The composition of these sequences is markedly different from LCD1 and LCD2 and contains a high fraction of hydrophobic residues (see Figure S1). The chimera proteins containing the LCD derived from elastin did not undergo phase separation under any investigated condition at room temperature, despite the high number of hydrophobic residues (Figure S5). However, we observed phase transition at higher temperatures, in agreement with the literature ${ }^{27}$ (Figure S7).

Moreover, we analyzed the propensity of the molecular adhesives to form intermolecular $\beta$-sheet structures. In particular, we calculated the propensity of LCD1 and LCD2 to convert into amyloid aggregates by using two common predictors of amyloid formation as a function of primary sequences, namely TANGO ${ }^{39,40}$ and AmylPred2. ${ }^{41}$ According to both algorithms, the sequences considered in this work have 
essentially no propensity to form $\beta$-sheet structures (Figure S8).

Overall, these results show that the molecular adhesives are capable of inducing attractive protein-protein interactions that in turn promote phase separation and the formation of proteinrich droplets. Such attractive interactions involve nonpolar, polar, and electrostatic forces, which can be tuned by $\mathrm{pH}$ and salt concentration, and are not based exclusively on a generic hydrophobic effect.

From Reversible Liquid Droplets to Irreversible Aggregates. The phase separation of our chimera proteins generates protein-rich liquid droplets that are highly dynamic and undergo coalescence (Figure 3A). We tested the

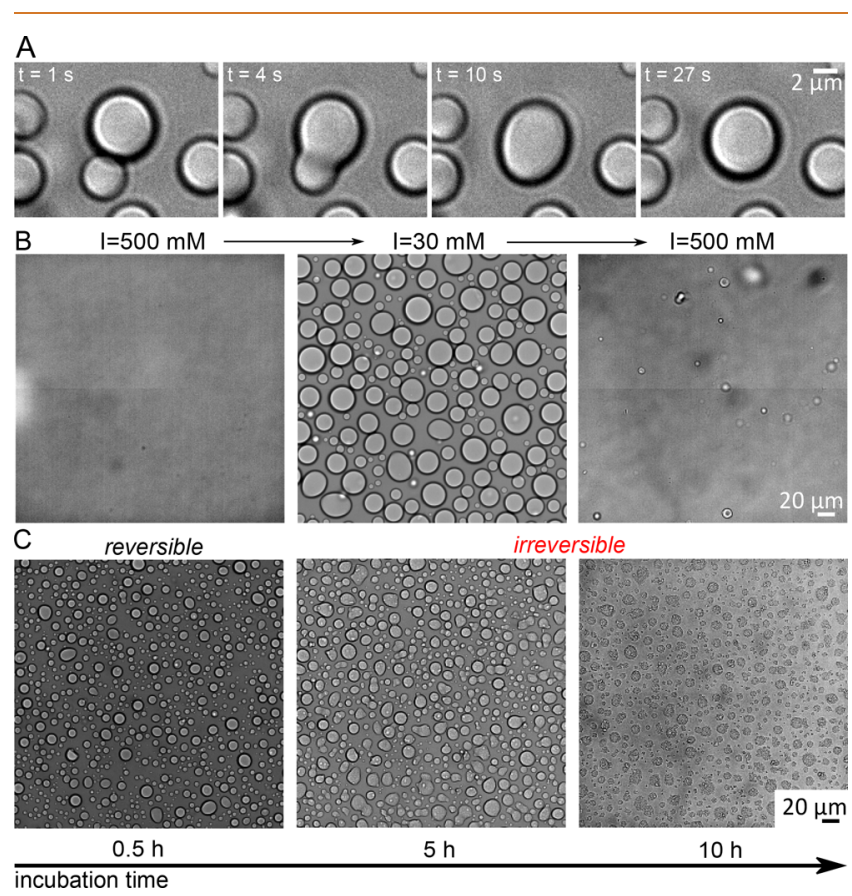

Figure 3. Maturation of reversible protein droplets into aggregates. (A) Coalescence of the liquid protein-rich droplets of $20 \mu \mathrm{M}$ AKLCD2 in $50 \mathrm{mM}$ Tris buffer at $\mathrm{pH}$ 8.0. (B) Reversibility of the phase separation of $20 \mu \mathrm{M}$ AK-LCD2 in $50 \mathrm{mM}$ Tris buffer at $\mathrm{pH}$ 8.5. The ionic strength was initially decreased from $500 \mathrm{mM}$ to 30 $\mathrm{mM}$ and then increased from $30 \mathrm{mM}$ to $500 \mathrm{mM}$. (C) During incubation time, the droplets shown in panel (A) evolve into solidified particles that are irreversible and maintain a spherical shape.

reversibility of this phase separation by varying salt concentration at $\mathrm{pH} 6.0$ for AK-LCD1 and $\mathrm{pH} 8.5$ for AKLCD2. We observed that the phase transition can be induced at low-salt concentration and can be reverted by increasing the ionic strength, thereby indicating that this process is reversible (Figure 3B). Notably, during incubation at room temperature over several hours, we observed the maturation of these liquid droplets into irreversible structures that do not coalesce and maintain the shape of the droplets (Figure 3C).

Similar observations have been made with several other peptides and proteins which have been shown to form protein crystals and other supramolecular structures via generation of metastable clusters consisting of dense liquid. ${ }^{42,43}$ Examples include both short peptides such as diphenylalanine ${ }^{44}$ and globular proteins like sickle-cell hemoglobin. ${ }^{45}$ It is becoming increasingly evident that proteins which undergo liquid-liquid phase separation have a high propensity to form aggregates via this two-step nucleation. For instance, disordered regions of key ribonucleoprotein (RNP) granule components and the full-length granule protein hnRNPAl have been shown to produce dynamic liquid droplets which mature to more stable states and form fibrous structures. ${ }^{46}$ Moreover, single point mutations of the fusion sarcoma protein (FUS) induce the maturation of dense liquid droplets into amyloid structures which have been associated with amyotrophic lateral sclerosis (ALS) disease. ${ }^{30,47}$ Our results, together with these previous observations, indicate that molecular adhesives based on LCDs have the generic potential to trigger controlled phase transition of soluble proteins into crystal and solid protein particles via formation of a dense liquid phase that matures over time. This understanding creates an attractive possibility to control the composition, size, and biophysical properties of the final structures by modulating the self-assembling process during early stages, when the protein-rich phase is dynamic.

Protein Particles Are Active Porous Structures and Release Soluble Proteins over Time. Before exploring the possibility of exploiting the initial dynamic state of the proteinrich phase to tune the composition of the aggregates, we first verified that the chimera proteins maintain their activity in the aggregated state by measuring the enzymatic activity of $\mathrm{AK}$ chimera proteins and the fluorescence of GFP chimera proteins. We observed that the aggregates of the GFP chimera proteins exhibit fluorescence, thereby indicating that GFP maintains its native activity. We then measured the activity of $\mathrm{AK}$ in the protein-rich phase by adding the corresponding substrate into a solution of $\mathrm{AK}$ protein particles and monitoring the formation of the fluorescent product by epifluorescence microscopy (Figure 4A). We tested both liquid droplets generated during the early stages of the process and mature solid aggregates formed over longer incubation times. For both structures, the images show the co-localization of the fluorescence intensity within the protein-rich phases, thereby indicating that the chimera proteins self-assemble into aggregates that retain their native activity (Figure 4A and Figure S9). We note that the protein particles exhibit similar activities to those of the soluble proteins at concentrations that are around 200-fold lower (Figure S10). Such decrease of the enzymatic activity in the aggregate state can be likely explained by the reduced amount of accessible active sites of the enzyme.

We observed that an increase of the salt concentration accelerates the conversion of the liquid droplets into solid particles and decreases the activity of the aggregates (Figure S11), suggesting that the control of the kinetics of the two-step nucleation process is important to regulate the activity of the final structures. Moreover, we performed a slow annealing experiment in which we let the solidified droplets equilibrate at different salt concentrations, either in steps or directly at 500 $\mathrm{mM} \mathrm{NaCl}$, and in both cases we did not observe any significant dissolution of the droplets (Figure S12).

Despite this decrease in activity, these results demonstrate the possibility to generate active protein particles that can potentially work as microbioreactors. To do so, these protein aggregates should exhibit a high level of porosity, thereby allowing the diffusion of soluble reagents and products into and out of these structures. We investigated this feature by monitoring the diffusion of a fluorescent probe, namely the dye Thioflavin $\mathrm{T}(\mathrm{ThT})$ in the presence of such aggregates. We observed that this molecular rotor enhances its fluorescence inside the protein-rich phase, either because of the increased 
A
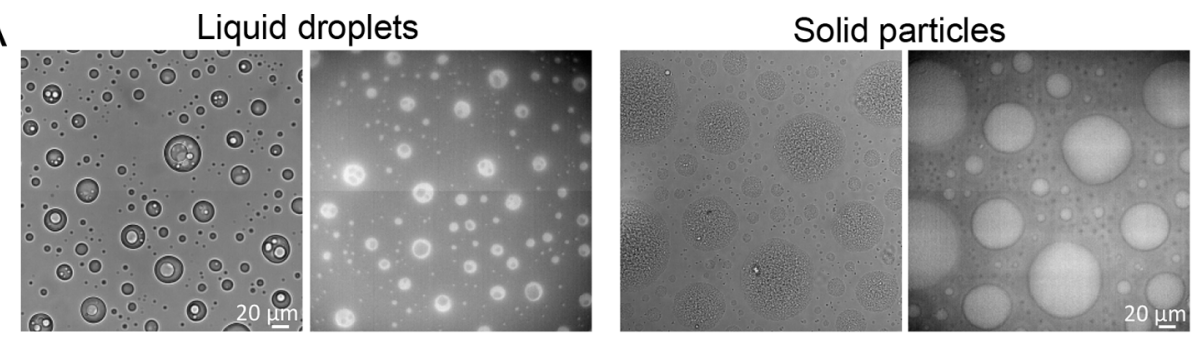

B
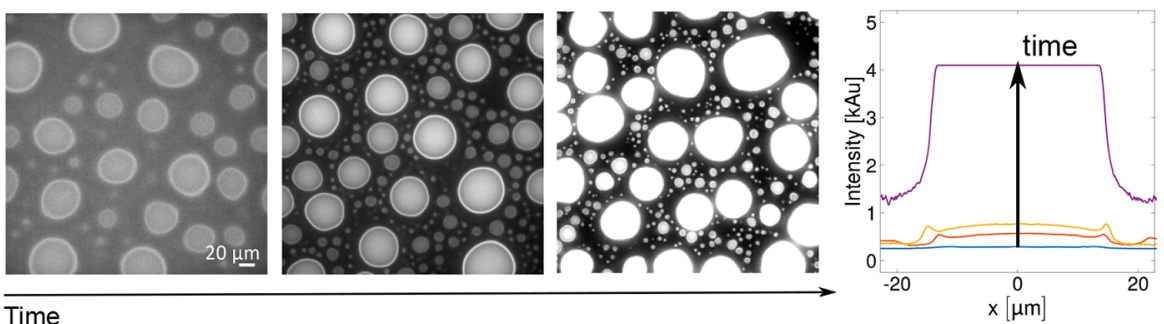

$$
\text { Time }
$$
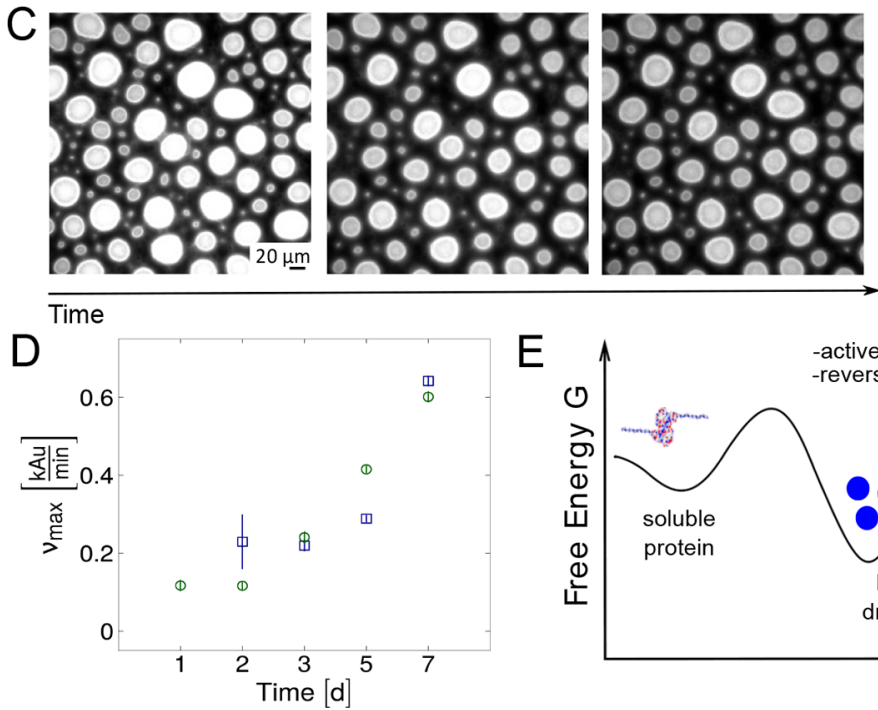

$\mathrm{E}$

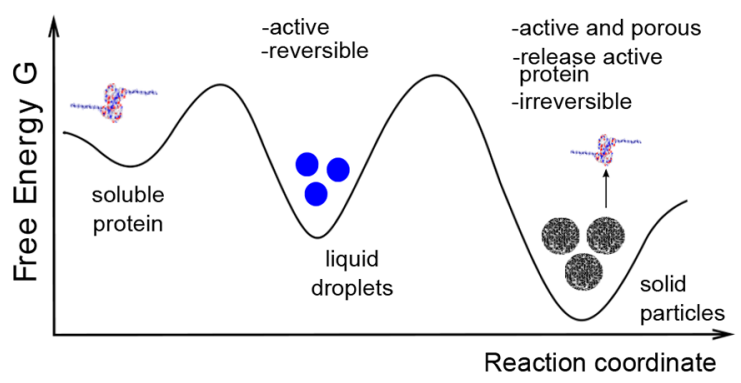

Figure 4. Protein particles are active porous structures and release soluble proteins over time. (A) Bright-field (left) and fluorescence microscopy (right) images of liquid droplets and solid particles of the chimera protein AK-LCD2. The formation of the product of the enzymatic reaction is monitored by recording fluorescence emission at $587 \mathrm{~nm}$ after excitation at $535 \mathrm{~nm}$. (B-C) Diffusion of the fluorescent probe ThT into and out of the solid aggregates of the chimera protein AK-LCD2. (B) Increase of the fluorescent intensity inside the aggregates over $40 \mathrm{~min}$ after introduction of ThT into the solution. From bottom to top, the curves represent data acquired at $0,5, \mathbf{1 0}$, and $20 \mathrm{~min}$ after the addition of ThT. (C) Decrease of the fluorescence intensity inside protein aggregates that have been pre-equilibrated for $2 \mathrm{~h}$ in a ThT solution and then introduced into a ThT-free buffer. From top to bottom, curves represent data acquired at 0, 10, 20, 40, 60, and 120 min after buffer exchange. (D) Enzymatic activity of the supernatant from a solution containing particles formed of protein AKLCD1 over time. Error bars indicate the two-sided 95\% confidences interval for the measured rate. Two independent sets of data are indicated in blue and green colors. In all the experiments, AK-LCD2 particles have been generated from a $20 \mu \mathrm{M}$ protein solution at $\mathrm{pH} 8.5$ containing no salt. (E) Schematic drawing of the two-step mechanism leading to the formation of protein aggregates from monomeric solutions via the formation of dense protein liquid state.

viscosity or because of interactions with proteins at high concentration, and can therefore be used to stain both the liquid droplets and the protein particles. We exploited this feature to monitor the diffusion of ThT into and out of the particles of AK-LCD2 by epi-fluorescence microscopy. To this aim, ThT was introduced into a solution of the protein particles, and the fluorescence profiles along the cross-section of protein aggregates were monitored over time (Figure 4B). In a second experiment, protein aggregates were preequilibrated with a solution containing ThT and incubated in a buffer without ThT, and the release of ThT into the supernatant was recorded over time by monitoring the fluorescence profile inside the protein particles (Figure 4C).
The two experiments revealed that the dye is capable of diffusing both into and out of the protein structures, thereby indicating that the protein particles are permeable structures.

Next, we tested whether or not the aggregates of AK-LCD2 release active soluble proteins over time. We washed the droplets 10 times with the assay buffer to remove any soluble proteins and exchanged the supernatant with a solution containing an excess of substrate of AK (see Materials and Methods and Figure S13). We then measured the activity of the supernatant by fluorescence spectroscopy over several days. The fluorescence intensity is proportional to the concentration of the product of the enzymatic reaction and therefore to the concentration of the soluble enzyme in solution. We observed 

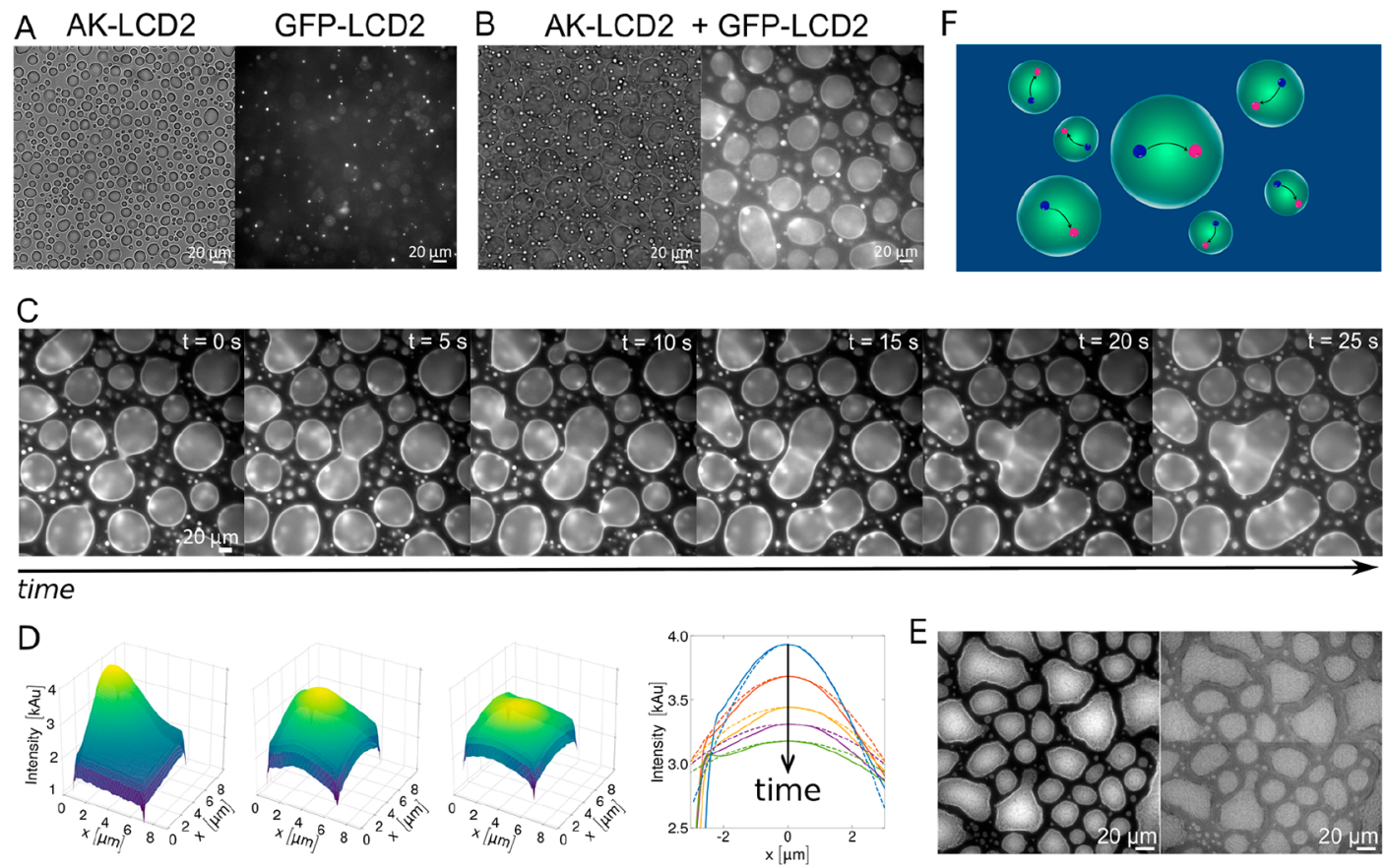

Figure 5. Multifunctional materials comprised of multiple chimeric proteins. (A) Microscope images of droplets formed from $20 \mu \mathrm{M}$ AKLCD2 (left) and $8 \mu \mathrm{M}$ GFP-LCD2 (right) solutions in $50 \mathrm{mM}$ Tris buffer at $\mathrm{pH}$ 8.5. (B) Bright field (left) and epi-fluorescence (right) images of the mixture of the two solutions in panel (A), showing the incorporation of the fluorescent GFP-LCD2 droplets into the larger AKLCD2 droplets. (C) After incorporation, the GFP-LCD2 droplets merge into the AK-LCD2 structures and lose their identity, thereby generating a homogeneous phase. (D) Time evolution of 3-D fluorescence profiles of GFP-LCD2 chimera proteins diffusing into the AKLCD2 matrix. The profiles have been extracted from images acquired at 0, 10, and $20 \mathrm{~s}$ after incorporation of the GFP-LCD2 droplet into the AK-LCD2 structures. The right-most plot shows the decrease of the fluorescence intensity along the cross section of the GFP-LCD2 droplet at 5, 10, 15, 20, and $25 \mathrm{~s}$ after the incorporation of one GFP-LCD2 droplet into one AK-LCD2 droplet. The dotted lines represent simulations based on Fick's diffusion law. (E) Fluorescent images showing the activity of the bifunctional AK-LCD2/GFP-LCD2 structures: fluorescence emission of GFP (left) and of the product of the enzymatic reactions catalyzed by AK (right). (F) Schematic drawing of the microreactors (Figure 4A) and multifunctional materials (panel E) developed in this work.

an increase of the fluorescence intensity over time (Figure 4D), indicating the release of soluble AK chimera proteins. Overall, these results demonstrate that the chimera protein maintains activity in the aggregate state.

Multifunctional Biomaterials and Microbioreactors. Lastly, we demonstrated the possibility of incorporating multiple functions within a given particle by exploiting the dynamic behavior of the dense liquid phase (Figure 4E). We predicted that mixtures of different globular proteins conjugated with the same molecular adhesives would induce not only self-assembly of a given chimera (Figure 2) but also attractive interactions between chimera proteins with different globular domains. To test this, we generated liquid droplets from solutions containing either AK-LCD2 or GFP-LCD2 (Figure 5A). Interestingly, when we mixed these solutions containing the preformed liquid droplets, we initially observed similar particles that were generated from the solutions containing the individual proteins alone (Figure 5B). However, we observed the GFP-LCD2 droplets in the mixture becoming incorporated into the larger AK-LCD2 structures over time (Figure 5C). After incorporation, the GFP chimera droplets appeared to diffuse into the dynamic matrix of AK-LCD2 proteins, thereby losing their identity as shown by the timedependent fluorescence profiles of GFP-LCD2 droplets after their incorporation within the AK-LCD2 structures (Figure $5 \mathrm{C}, \mathrm{D})$. In order to interpret our data in a more quantitative way, we described the time evolution of the cross-sectional fluorescence by Fick's diffusion law $^{48}$ (Figure 5D). The comparison between model simulations and experimental data provided a diffusion coefficient of the GFP-LCD2 protein of $1.1 \times 10^{-12} \mathrm{~m}^{2} / \mathrm{s}$, which is about 1 order of magnitude smaller compared to the value measured in solution by DLS $\left(8.9 \times 10^{-12} \mathrm{~m}^{2} / \mathrm{s}\right)$. This result indicates that the viscosity of the protein-rich phase is indeed higher as compared to aqueous solutions, yet still sufficiently low to guarantee high molecular diffusion. After $1 \mathrm{~h}$, we observed an arrest of the diffusion process and the presence of a homogeneous structure containing two different proteins, which is preserved during the maturation into the solid-like aggregates. We verified that the function of both proteins is preserved within this structure by adding the reagents of the enzymatic reaction catalyzed by $\mathrm{AK}$ and simultaneously acquiring fluorescence images corresponding to both the GFP protein and the fluorescent product of the reaction (Figure $5 \mathrm{E}$ ).

Taken together, our results demonstrate that the molecular adhesives described in this work represent a promising strategy to bring together different proteins within the same architecture, thereby creating multifunctional biomaterials and microscale bioreactors exhibiting high concentrations of homogeneously dispersed reagents.

\section{CONCLUSIONS}

We have developed molecular adhesives based on disordered sequences of proteins found in nature associated with phase separation. We have demonstrated that the conjugation of globular proteins to these sequences induces a controlled self- 
assembly into supramolecular structures via formation of dense liquid phases that mature over time into solid-like aggregates. The molecular adhesives enable one to induce specific attractive interactions with sequences that are rich in specific amino acids. Such attractive interactions include nonpolar, polar, and electrostatic forces and are not based on general hydrophobicity. The globular domains within our fusion proteins maintain their activity in the aggregated state. We have shown that this strategy can be applied to develop permeable protein materials that release active proteins over time. Moreover, we have demonstrated that the dynamic nature of the protein-rich phase during the early stages of the maturation process, together with the specific interactions induced by the LCDs, can be exploited to control the composition and the size of the final aggregates. Furthermore, we have shown that the molecular adhesives can bring together different functionalities within the same architecture. This technology, together with the control of the kinetics of the two-step nucleation process, leads to the generation of both static multifunctional biomaterials and dynamic, liquid microscale bioreactors.

\section{MATERIAL AND METHODS}

DNA Constructs and Protein Production. All DNA sequences were synthesized, codon optimized for expression in E. coli, and cloned into the pET-15b vector by Genewiz (NJ, US). LCDs from different native proteins (Dhh1 from Saccharomyces cerevisiae, AA 147 and AA 426-506; Dbp1 from S. cerevisiae, AA 1-155 and AA 552-618; elastin derived sequence (VHPGVG) ${ }_{25}$ ) was fused with either $E$. coli adenylate kinase (AK) or green fluorescence protein (GFP). The complete sequences of the fusion proteins used in this study are listed in Figure S1. Recombinant proteins were expressed in E. coli BL21-GOLD (DE3) cells. Bacterial cultures were induced at OD 0.7 with $0.5 \mathrm{mM}$ isopropyl D-thiogalactopyranoside (99\%, PanReac AppliChem) and grown for an additional $16 \mathrm{~h}$ at 20 or 37 ${ }^{\circ} \mathrm{C}$ in case of AK-LCD2. Recombinant proteins were purified using immobilized metal ion affinity chromatography (Chelating Sepharose, GE Healthcare) according to a standard protocol. As a final purification step, we performed size exclusion chromatography using a Superdex 75 26/600 on an ÄKTA Prime system (GE Healthcare) in $50 \mathrm{mM}$ Tris (pH 7.5) with $200 \mathrm{mM} \mathrm{NaCl}$ (AK-LCD1, GFP- LCD1, AK-Elastin) or $50 \mathrm{mM}$ Tris ( $\mathrm{pH} 8.5$ ) with $600 \mathrm{mM} \mathrm{NaCl}$ (AKLCD2) or $1 \mathrm{M} \mathrm{NaCl}$ (GFP-LCD2). The quality of the purified proteins was checked by SDS-PAGE electrophoresis (Figure S2) and SEC-MALS analysis (Figure S3). Proteins were concentrated to 20$30 \mathrm{mg} / \mathrm{mL}$, and aliquots were frozen and stored at $-20{ }^{\circ} \mathrm{C}$ until use. The fractions of charged residues (FCR), polar residues (FPR), and nonpolar residues (FNPR) were calculated according to Das et al. ${ }^{49}$ at the reference $\mathrm{pH}$ of 7.5. Charges at different $\mathrm{pH}$ values were estimated using Prot pI, and the pI was estimated via ExPASy.

Size Exclusion Chromatography Coupled with Multi-Angle Light Scattering (SEC-MALS). The quaternary state of all the fusion proteins was analyzed by injecting $30 \mathrm{ng}$ of protein into a Superdex 75 10/300 GL (GE Healthcare) size exclusion column assembled on an Agilent 1200 Series HPLC. The molecular weight of the fractionated proteins was measured in-line with an 18-angle light scattering detector (Dawn Heleos II, Wyatt).

Phase Diagrams and Characterization of Droplet Size, Stability, and Fusion Times. Phase diagrams were obtained by analyzing solutions at fixed protein concentration $(\sim 1 \mathrm{mg} / \mathrm{mL})$ at varying $\mathrm{pH}$ and salt concentrations by differential interference contrast (DIC) detection, using a $60 \times$ oil objective (CFI Plan Apo Lambda $60 \times$ Oil, Nikon) on an epi-fluorescence microscope (Eclipse Ti-E, Nikon). Images were collected $30 \mathrm{~min}$ after sample preparation using a CCD camera (Zyla sCMOS, Andor). All the measurements were performed in a 384-well plate (MatriPlate 384Well Plate, Glass Bottom, Brooks).
In order to determine the stability of the droplets, 300 images were taken every $2.5 \mathrm{~min}$ for $12.5 \mathrm{~h}$. In addition to DIC detection, the droplets were stained by Thioflavin $\mathrm{T}$ dye (ThT) and imaged using a $455 \mathrm{~nm}$ High power LED light source (LedHUB Light engine, Omicron) equipped with a filter with excitation $426-446 \mathrm{~nm}$ and emission 460-500 nm (CFP ET Filter set, Chroma Technology Corporation). Fusion events were monitored by $1 \mathrm{~s}$ time lapses.

Activity Assays. The activity of the adenylate kinase recombinant proteins was assessed using a standard fluorometric detection assay Abcam (ab211095). Fluorescence emission was detected at $587 \mathrm{~nm}$ after excitation at $535 \mathrm{~nm}$ using a microplate reader (CLARIOstar, BMG Labtech).

The fluorescence of GFP and GFP fusion proteins was measured by a epi-fluorescence microscope (Ti-E, Nikon) equipped with a 455 $\mathrm{nm}$ high power LED light source (LedHUB light engine, Omicron), a 455-490 nm excitation, and a 505-550 emission width filter set (EGFP ET Filter set, Chroma Technology Corporation).

Analysis of Diffusion Profiles. The cross-sectional fluorescence profiles of GFP-LCD2 droplets, which were acquired at different time points after the incorporation within the AK-LCD2 structures (Figure 5C,D), were analyzed according to Fick's diffusion law. This equation can be solved for an unsteady decay of a pulse as shown by Cussler ${ }^{50}$ leading to

$$
c=\frac{c_{0}}{\sqrt{4 \pi D t}} \mathrm{e}^{-z^{2} / 4 D t}
$$

where $c$ denotes the concentration of the protein, $D$ the diffusion coefficient, $t$ the time, $z$ the diffusion direction, and $c_{0}$ the initial concentration at $z$ equal to zero. Eq 1 was globally fit to the five experimental intensity profiles by minimizing a least-squares error function, assuming linear proportionality between the intensity and the concentration of GFP-LCD2.

The diffusion coefficient of the GFP-LCD2 protein is related to the viscosity of the protein-rich phase $(\eta)$ via the Stokes-Einstein relationship:

$$
D=\frac{k_{\mathrm{B}} T}{6 \pi \eta r}
$$

where $r$ denotes the hydrodynamic radius of the protein, $k_{\mathrm{B}}$ the Boltzmann constant, and $T$ the absolute temperature.

Dynamic Light Scattering. The diffusion coefficient of GFPLCD2 protein in solution and the average hydrodynamic radius of different protein solutions were measured by DLS on a Zetasizer instrument (Malvern, UK), working in backscattering mode at $173^{\circ}$ with a laser source of $633 \mathrm{~nm}$.

\section{ASSOCIATED CONTENT}

\section{S Supporting Information}

The Supporting Information is available free of charge on the ACS Publications website at DOI: 10.1021/acsnano.8b04304.

Supporting experimental data on the characterization of protein sequences and activity as well as of the phase separation process. Tables $\mathrm{S} 1$ and Figures S1-S13 (PDF)

\section{AUTHOR INFORMATION}

\section{Corresponding Author}

*E-mail: paolo.arosio@chem.ethz.ch.

ORCID ${ }^{\circ}$

Paolo Arosio: 0000-0002-2740-1205

\section{Author Contributions}

${ }^{\S}$ These authors contributed equally to this work.

Notes

The authors declare no competing financial interest. 


\section{ACKNOWLEDGMENTS}

The Arosio laboratory gratefully acknowledges financial support from the Swiss National Science Foundation (grant 205321_179055) and the Novartis Foundation for Biomedical and Biological Research.

\section{REFERENCES}

(1) Knowles, T. P. J.; Vendruscolo, M.; Dobson, C. M. The Amyloid State and Its Association with Protein Misfolding Diseases. Nat. Rev. Mol. Cell Biol. 2014, 15, 384-396.

(2) Maji, S. K.; Perrin, M. H.; Sawaya, M. R.; Jessberger, S.; Vadodaria, K.; Rissman, R. A.; Singru, P. S.; Nilsson, K. P. R.; Simon, R.; Schubert, D.; Eisenberg, D.; Rivier, J.; Sawchenko, P.; Vale, W.; Riek, R. Functional Amyloids as Natural Storage of Peptide Hormones in Pituitary Secretory Granules. Science 2009, 325, 328332.

(3) Fowler, D. M.; Koulov, A. V.; Balch, W. E.; Kelly, J. W. Functional Amyloid - from Bacteria to Humans. Trends Biochem. Sci. 2007, 32, 217-224.

(4) Blanco, L. P.; Evans, M. L.; Smith, D. R.; Badtke, M. P.; Chapman, M. R. Diversity, Biogenesis and Function of Microbial Amyloids. Trends Microbiol. 2012, 20, 66-73.

(5) Knowles, T. P. J.; Mezzenga, R. Amyloid Fibrils as Building Blocks for Natural and Artificial Functional Materials. Adv. Mater. 2016, 28, 6546-6561.

(6) Wei, G.; Su, Z.; Reynolds, N. P.; Arosio, P.; Hamley, I. W.; Gazit, E.; Mezzenga, R. Self-Assembling Peptide and Protein Amyloids: From Structure to Tailored Function in Nanotechnology. Chem. Soc. Rev. 2017, 46, 4661-4708.

(7) Mahler, A.; Reches, M.; Rechter, M.; Cohen, S.; Gazit, E. Rigid, Self-Assembled Hydrogel Composed of a Modified Aromatic Dipeptide. Adv. Mater. 2006, 18, 1365-1370.

(8) Saiani, A.; Mohammed, A.; Frielinghaus, H.; Collins, R.; Hodson, N.; Kielty, C. M.; Sherratt, M. J.; Miller, A. F. Self-Assembly and Gelation Properties of $\alpha$-Helix versus $\beta$-Sheet Forming Peptides. Soft Matter 2009, 5, 193-202.

(9) Lamm, M. S.; Rajagopal, K.; Schneider, J. P.; Pochan, D. J. Laminated Morphology of Nontwisting $\beta$-Sheet Fibrils Constructed via Peptide Self-Assembly. J. Am. Chem. Soc. 2005, 127, 1669216700 .

(10) Cheng, G.; Castelletto, V.; Jones, R. R.; Connon, C. J.; Hamley, I. W. Hydrogelation of Self-Assembling RGD-Based Peptides. Soft Matter 2011, 7, 1326-1333.

(11) Aggeli, A.; Bell, M.; Carrick, L. M.; Fishwick, C. W. G.; Harding, R.; Mawer, P. J.; Radford, S. E.; Strong, A. E.; Boden, N. PH as a Trigger of Peptide $\beta$-Sheet Self-Assembly and Reversible Switching between Nematic and Isotropic Phases. J. Am. Chem. Soc. 2003, 125, 9619-9628.

(12) Yokoi, H.; Kinoshita, T.; Zhang, S. Dynamic Reassembly of Peptide RADA16 Nanofiber Scaffold. Proc. Natl. Acad. Sci. U. S. A. 2005, 102, 8414-8419.

(13) Cui, H. G.; Webber, M. J.; Stupp, S. I. Self-Assembly of Peptide Amphiphiles: From Molecules to Nanostructures to Biomaterials. Biopolymers 2010, 94, 1-18.

(14) Pashuck, E. T.; Duchet, B. J. R.; Hansel, C. S.; Maynard, S. A.; Chow, L. W.; Stevens, M. M. Controlled Sub-Nanometer Epitope Spacing in a Three-Dimensional Self-Assembled Peptide Hydrogel. ACS Nano 2016, 10, 11096-11104.

(15) Parmar, P. A.; Chow, L. W.; St-Pierre, J. P.; Horejs, C. M.; Peng, Y. Y.; Werkmeister, J. A.; Ramshaw, J. A. M.; Stevens, M. M. Collagen-Mimetic Peptide-Modifiable Hydrogels for Articular Cartilage Regeneration. Biomaterials 2015, 54, 213-225.

(16) Shimanovich, U.; Efimov, I.; Mason, T. O.; Flagmeier, P.; Buell, A. K.; Gedanken, A.; Linse, S.; Akerfeldt, K. S.; Dobson, C. M.; Weitz, D. A.; Knowles, T. P. J. Protein Microgels from Amyloid Fibril Networks. ACS Nano 2015, 9, 43-51.
(17) Azuma, Y.; Zschoche, R.; Tinzl, M.; Hilvert, D. Quantitative Packaging of Active Enzymes into a Protein Cage. Angew. Chem., Int. Ed. 2016, 55, 1531-1534.

(18) Huang, X.; Li, M.; Green, D. C.; Williams, D. S.; Patil, A. J.; Mann, S. Interfacial Assembly of Protein-Polymer Nano-Conjugates into Stimulus-Responsive Biomimetic Protocells. Nat. Commun. 2013, 4, 2239.

(19) Shen, Y.; Posavec, L.; Bolisetty, S.; Hilty, F. M.; Nyström, G.; Kohlbrecher, J.; Hilbe, M.; Rossi, A.; Baumgartner, J.; Zimmermann, M. B.; Mezzenga, R. Amyloid Fibril Systems Reduce, Stabilize and Deliver Bioavailable Nanosized Iron. Nat. Nanotechnol. 2017, 12, 642-647.

(20) Bolisetty, S.; Mezzenga, R. Amyloid-Carbon Hybrid Membranes for Universal Water Purification. Nat. Nanotechnol. 2016, 11, 365-371.

(21) Hartgerink, J. D.; Beniash, E.; Stupp, S. I. Self-Assembly and Mineralization of Peptide-Amphiphile Nanofibers. Science 2001, 294, 1684-1688.

(22) Hudalla, G. A.; Sun, T.; Gasiorowski, J. Z.; Han, H. F.; Tian, Y. F.; Chong, A. S.; Collier, J. H. Gradated Assembly of Multiple Proteins into Supramolecular Nanomaterials. Nat. Mater. 2014, 13, 829-836.

(23) Moon, J. J.; Suh, H.; Bershteyn, A.; Stephan, M. T.; Liu, H.; Huang, B.; Sohail, M.; Luo, S.; Ho Um, S.; Khant, H.; Goodwin, J. T.; Ramos, J.; Chiu, W.; Irvine, D. J. Interbilayer-Crosslinked Multilamellar Vesicles as Synthetic Vaccines for Potent Humoral and Cellular Immune Responses. Nat. Mater. 2011, 10, 243-251.

(24) Adler-Abramovich, L.; Gazit, E. The Physical Properties of Supramolecular Peptide Assemblies: From Building Block Association to Technological Applications. Chem. Soc. Rev. 2014, 43, 6881-6893.

(25) Zhou, X. M.; Entwistle, A.; Zhang, H.; Jackson, A. P.; Mason, T. O.; Shimanovich, U.; Knowles, T. P. J.; Smith, A. T.; Sawyer, E. B.; Perrett, S. Self-Assembly of Amyloid Fibrils That Display Active Enzymes. ChemCatChem 2014, 6, 1961-1968.

(26) Simon, J. R.; Carroll, N. J.; Rubinstein, M.; Chilkoti, A.; López, G. P. Programming Molecular Self-Assembly of Intrinsically Disordered Proteins Containing Sequences of Low Complexity. Nat. Chem. 2017, 9, 509-515.

(27) Quiroz, F. G.; Chilkoti, A. Sequence Heuristics to Encode Phase Behaviour in Intrinsically Disordered Protein Polymers. Nat. Mater. 2015, 14, 1164-1171.

(28) Elbaum-Garfinkle, S.; Kim, Y.; Szczepaniak, K.; Chen, C. C.; Eckmann, C. R.; Myong, S.; Brangwynne, C. P. The Disordered P Granule Protein LAF-1 Drives Phase Separation into Droplets with Tunable Viscosity and Dynamics. Proc. Natl. Acad. Sci. U. S. A. 2015, 112, 7189-7194.

(29) Wei, M. T.; Elbaum-Garfinkle, S.; Holehouse, A. S.; Chen, C. C.; Feric, M.; Arnold, C. B.; Priestley, R. D.; Pappu, R. V.; Brangwynne, C. P. Phase Behaviour of Disordered Proteins Underlying Low Density and High Permeability of Liquid Organelles. Nat. Chem. 2017, 9, 1118-1125.

(30) Patel, A.; Lee, H. O.; Jawerth, L.; Maharana, S.; Jahnel, M.; Hein, M. Y.; Stoynov, S.; Mahamid, J.; Saha, S.; Franzmann, T. M.; Pozniakovski, A.; Poser, I.; Maghelli, N.; Royer, L. A.; Weigert, M.; Myers, E. W.; Grill, S.; Drechsel, D.; Hyman, A. A.; Alberti, S. A Liquid-to-Solid Phase Transition of the ALS Protein FUS Accelerated by Disease Mutation. Cell 2015, 162 (5), 1066-1077.

(31) Thandapani, P.; O'Connor, T. R.; Bailey, T. L.; Richard, S. Defining the RGG/RG Motif. Mol. Cell 2013, 50, 613-623.

(32) Mugler, C. F.; Hondele, M.; Heinrich, S.; Sachdev, R.; Vallotton, P.; Koek, A. Y.; Chan, L. Y.; Weis, K. ATPase Activity of the DEAD-Box Protein Dhh1 Controls Processing Body Formation. eLife 2016, 5, e18746.

(33) Shin, Y.; Brangwynne, C. P. Liquid Phase Condensation in Cell Physiology and Disease. Science 2017, 357, eaaf4382.

(34) Brangwynne, C. P.; Tompa, P.; Pappu, R. V. Polymer Physics of Intracellular Phase Transitions. Nat. Phys. 2015, 11, 899. 
(35) Malinovska, L.; Kroschwald, S.; Alberti, S. Protein Disorder, Prion Propensities, and Self-Organizing Macromolecular Collectives. Biochim. Biophys. Acta, Proteins Proteomics 2013, 1834, 918-931.

(36) Schmidt, H. B.; Görlich, D. Transport Selectivity of Nuclear Pores, Phase Separation, and Membraneless Organelles. Trends Biochem. Sci. 2016, 41, 46-61.

(37) Protter, D. S. W.; Rao, B. S.; Van Treeck, B.; Lin, Y.; Mizoue, L.; Rosen, M. K.; Parker, R. Intrinsically Disordered Regions Can Contribute Promiscuous Interactions to RNP Granule Assembly. Cell Rep. 2018, 22, 1401-1412.

(38) Qamar, S.; Wang, G. Z.; Randle, S. J.; Ruggeri, F. S.; Varela, J. A.; Lin, J. Q.; Phillips, E. C.; Miyashita, A.; Williams, D.; Ströhl, F.; Meadows, W.; Ferry, R.; Dardov, V. J.; Tartaglia, G. G.; Farrer, L. A.; Kaminski Schierle, G. S.; Kaminski, C. F.; Holt, C. E.; Fraser, P. E.; Schmitt-Ulms, G.; et al. FUS Phase Separation Is Modulated by a Molecular Chaperone and Methylation of Arginine Cation- $\pi$ Interactions. Cell 2018, 173, 720-734.

(39) Fernandez-Escamilla, A. M.; Rousseau, F.; Schymkowitz, J.; Serrano, L. Prediction of Sequence-Dependent and Mutational Effects on the Aggregation of Peptides and Proteins. Nat. Biotechnol. 2004, 22, 1302-1306.

(40) Linding, R.; Schymkowitz, J.; Rousseau, F.; Diella, F.; Serrano, L. A Comparative Study of the Relationship between Protein Structure and $\beta$-Aggregation in Globular and Intrinsically Disordered Proteins. J. Mol. Biol. 2004, 342, 345-353.

(41) Tsolis, A. C.; Papandreou, N. C.; Iconomidou, V. A.; Hamodrakas, S. J. A Consensus Method for the Prediction of "Aggregation-Prone" Peptides in Globular Proteins. PLoS One 2013, 8 , e54175.

(42) Vekilov, P. G. Nucleation of Crystals in Solution. AIP Conf. Proc. 2010, 1270, 60-77.

(43) Gliko, O.; Neumaier, N.; Pan, W.; Haase, I.; Fischer, M.; Bacher, A.; Weinkauf, S.; Vekilov, P. G. A Metastable Prerequisite for the Growth of Lumazine Synthase Crystals. J. Am. Chem. Soc. 2005, 127, 3433-3438.

(44) Levin, A.; Mason, T. O.; Adler-Abramovich, L.; Buell, A. K.; Meisl, G.; Galvagnion, C.; Bram, Y.; Stratford, S. A.; Dobson, C. M.; Knowles, T. P. J.; Gazit, E. Ostwalds Rule of Stages Governs Structural Transitions and Morphology of Dipeptide Supramolecular Polymers. Nat. Commun. 2014, 5, 5219.

(45) Galkin, O.; Pan, W.; Filobelo, L.; Hirsch, R. E.; Nagel, R. L.; Vekilov, P. G. Two-Step Mechanism of Homogeneous Nucleation of Sickle Cell Hemoglobin Polymers. Biophys. J. 2007, 93, 902-913.

(46) Lin, Y.; Protter, D. S.; Rosen, M. K.; Parker, R. Formation and Maturation of Phase-Separated Liquid Droplets by RNA-Binding Proteins. Mol. Cell 2015, 60, 208-219.

(47) Mateju, D.; Franzmann, T. M.; Patel, A.; Kopach, A.; Boczek, E. E.; Maharana, S.; Lee, H. O.; Carra, S.; Hyman, A. A.; Alberti, S. An Aberrant Phase Transition of Stress Granules Triggered by Misfolded Protein and Prevented by Chaperone Function. EMBO J. 2017, 36, $1669-1687$.

(48) Fick, A. V. On Liquid Diffusion. London, Edinburgh, Dublin Philos. Mag. J. Sci. 1855, 10, 30-39.

(49) Das, R. K.; Ruff, K. M.; Pappu, R. V. Relating Sequence Encoded Information to Form and Function of Intrinsically Disordered Proteins. Curr. Opin. Struct. Biol. 2015, 32, 102-112.

(50) Cussler, E. L. Diffusion in Dilute Solutions. In Diffusion mass transfer in fluid systems; Cambridge University Press: Cambridge, UK, 2009; pp 13-49. 